\title{
Treatment of Botulinum Toxin Type A in Parotid Fistula after Face Lifting
}

\section{Min Su Jung, Byeong Ho Lee, Joo Hyun Kim, Seong Hoon Park, Duk Kyun Ahn, Hii Sun Jeong, In Suck Suh}

Department of Plastic and Reconstructive Surgery, KangNam Sacred Heart Hospital, College of Medicine, Hallym University, Seoul, Korea

This article was presented as a free paper at the 2014 international meeting of the Korean society for aesthetic plastic surgery on April 26-27, 2014.

No potential conflict of interest relevant to this article was reported.
Botulinum toxin type A has an inhibitory action not only on neuromuscular junctions, but also postganglionic sympathetic and cholinergic autonomic parasympathetic acetylcholine release at the secretary end of the salivary gland. Use of botulinum toxin to treat sialorrhea was first reported in 1997 by Bushara. Parotid duct or gland injuries with parotid fistula are uncommon but troublesome complications of surgical trauma. Here, we report two patients with constant leakage of serous fluid and a swelling cheek after facelift surgery. Each patient underwent an amylase test, starch iodine test, and sialography. After diagnosis of parotid fistula, a total of 50 units botulinum toxin was injected into the parotid gland. Facial bandage, scopolamine, and minimizing temporomandibular joint motion were instructed. Leakage volume decreased gradually, and symptoms subsided within 2 weeks. Neither functional problems nor complications occurred. In conclusion, a parotid fistula after facial surgery can be treated effectively with botulinum toxin; this treatment option should be considered before proceeding with invasive surgical treatment.

Keywords Botulinum toxin A, Facelift, Fistula, Parotid gland

\section{INTRODUCTION}

Increasing numbers of Koreans, especially elderly Koreans, have been undergoing facelift surgery for rejuvenation purposes. Although uncommon, troublesome complications such as parotid duct injuries or parotid gland fistulas can occur as a result of facelift surgery. There are a few reported cases of iatrogenic surgical complications of the parotid gland during facelift surgery [1-3]. Needle aspiration, pressure dressings, and anticholinergics are commonly used to treat parotid fistulas [4,5]. A persistent parotid fistula is usually treated by invasive surgical procedure; however, facelift patients tend to receive less invasive procedures due to cos-

Received: Jun 20, 2014 Accepted: Jun 23, 2014

Correspondence: In Suck Suh Department of Plastic and Reconstructive Surgery, KangNam Sacred Heart Hospital, College of Medicine, Hallym University, 1 Singillo, Yongdeongpo-gu, Seoul 150-071, Korea.

E-mail: sismdps@chol.com

Copyright @ 2014 The Korean Society for Aesthetic Plastic Surgery.

This is an Open Access article distributed under the terms of the Creative Commons Attribution Non-Commercial License (http://creativecommons.org/licenses/by-nc/3.0/) which permits unrestricted non-commercial use, distribution, and reproduction in any medium, provided the original work is properly cited. www.e-aaps.org metic reasons. Here, we report two cases of parotid fistula after facelift surgery successfully treated with botulinum toxin type A. Botulinum toxin decreases salivation though inhibition of acetylcholine release [5] and is therefore suitable for treating a parotid fistula after a facelift procedure.

\section{CASE REPORTS}

\section{Case 1}

A 58-year old woman was referred to our department of plastic surgery 7 days after facelift surgery and neck liposuction performed at a private clinic. The patient complained of immediate post-operative salivary leakage (about $15 \mathrm{~mL}$ to $20 \mathrm{~mL}$ per day) in the right posterior auricle area, especially when chewing food, and swelling around the right parotid gland. Despite 6 days of pressure dressing and hemovac insertion, her symptoms showed no improvement prior to this visit. On physical examination, she had an asymmetric face contour, with severe swelling around the right mandible angle (Fig. 1A). Amylase concentration of the salivary leakage could not be determined as it was higher than the detection limits of the assay $(>65,000 \mathrm{U} / \mathrm{mL})$. Starch-iodine test revealed that her secretion changed from purple to light brown, in- 

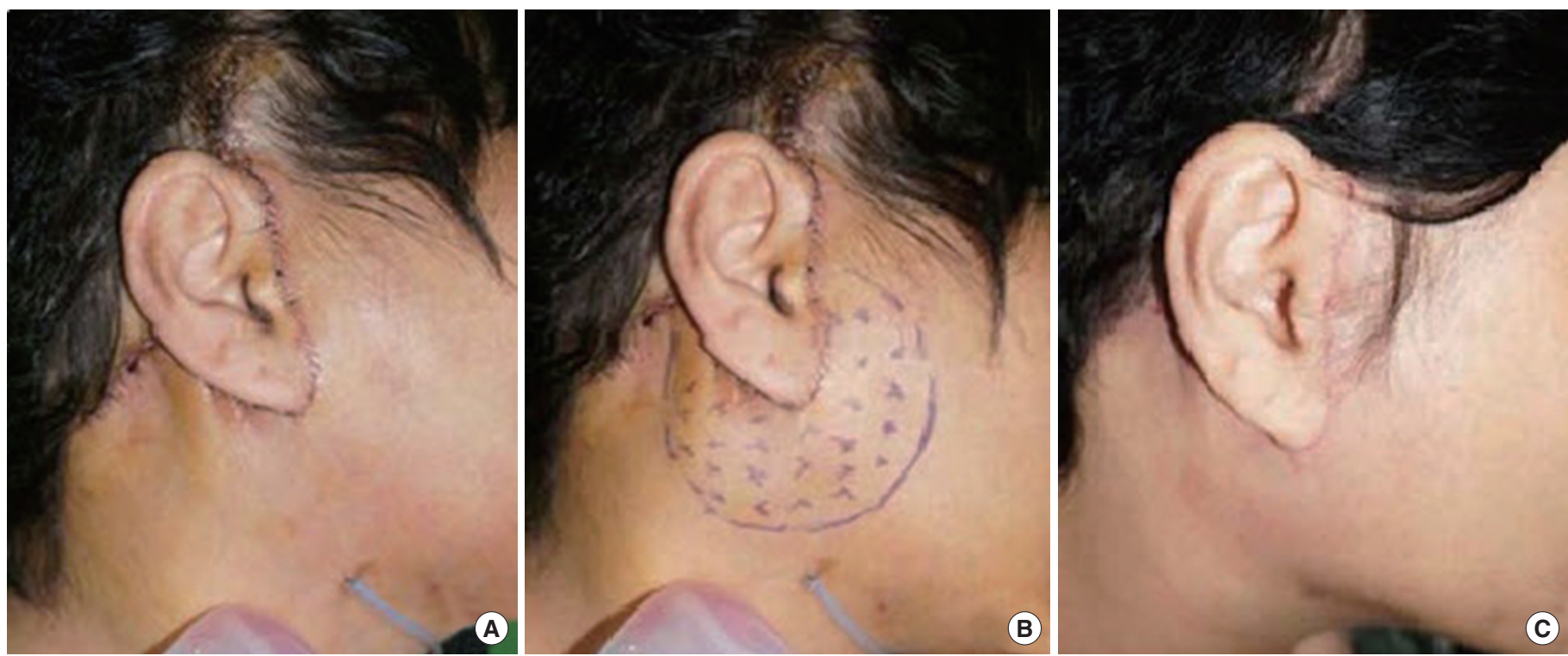

Fig. 1. (A) Lateral view showing asymmetric face contour with deviation to the right because of severe swelling around the right mandible angle. (B) Designed lateral view before botulinum toxin injection. (C) Nine days after botulinum toxin injection, both salivary leakage and right cheek swelling had resolved completely.

dicating that the excessive secretion was mainly due to abnormality in salivary function. Sialography demonstrated that the right Stensen's duct was not damaged. We injected intraglandular botulinum toxin type A (Botulex ${ }^{\circledR}$, Hugel, Chuncheon, Korea) into the right parotid gland. A total of 50 units of botulinum toxin was diluted in $3 \mathrm{~mL}$ of normal saline. Two units of botulinum toxin were injected for each interinjection distance of $1 \mathrm{~cm}$ (Fig. 1B). We also prescribed the anticholinergic drug, scopolamine, and placed a facial bandage over the patient's face to compress the injected site. Patient was instructed to eat rice porridge and minimize temporomandibular joint motion. Follow-up was performed every 3 days. On the first follow-up visit, leakage volume had decreased significantly. Both salivary leakage and swelling of the right cheek resolved completely within 10 days (Fig. 1C). No side-effects were noted. To date, 3 months after the botulinum toxin injection, the patient is free of recurrence.

\section{Case 2}

A 52-year old woman was referred to our department due to 20 $-25 \mathrm{~mL}$ salivary leakage at the right periauricular area every day. She had undergone facelift surgery 1 month prior at a private clinic, and experienced immediate post-operative salivary leakage and swelling of the right mandibular angle. No improvement was seen after hemovac insertion or pressure dressing. Her right eye and right mandible were slightly swollen and bruised (Fig. 2A). She had no symptoms of right facial nerve palsy. Amylase concentration of the salivary leakage could not be determined as it was higher than the detection limits of the assay $(>65,000 \mathrm{U} / \mathrm{mL})$. In the starch-iodine test, the sample changed color from purple to light brown, proving salivary reaction. Sialography of the right parotid gland demonstrated that Stensen's duct was intact. We found that the patient actually had salivary leakage of $100 \mathrm{~mL}$ a day. Hence, we decided to maintain the hemovac drain, continue pressure dressing, and prescribed scopolamine for 2 weeks to reduce salivary secretion before injecting botulinum toxin, because the patient did not want botulinum toxin injection or a surgical procedure immediately. A total of 50 units of intraglandular botulinum toxin type A (Botulex ${ }^{\circledR}$, Hugel, Chuncheon, Korea) was diluted in $3 \mathrm{~mL}$ of normal saline and injected into the right parotid gland after 2 weeks. Two units were injected for each interinjection distance of $1 \mathrm{~cm}$ (Fig. 2B). The patient was instructed to continue taking scopolamine, eat rice porridge, and limit temporomandibular joint motion. Facial bandage was placed over her head and face, including the right parotid region. We checked her symptoms every 3 days. Two weeks after the injection, she was finally free of discomfort (Fig. 2C). To date, 6 months after botulinum toxin therapy, the patient is free of recurrence.

\section{DISCUSSION}

Facelift surgery, known as rhytidectomy, is a common cosmetic surgery used to provide a more youthful facial appearance. Various complications of rhytidectomy include hematoma, nerve injuries, skin necrosis, hair loss, infection, and parotid gland duct injury. Incidence of parotid gland duct injury has been increasing as surgical methods for rhytidectomy have been becoming more aggressive. Parotid duct injury may result in a parotid fistula, which is a localized, subcutaneous cavity containing saliva. Disruption of 

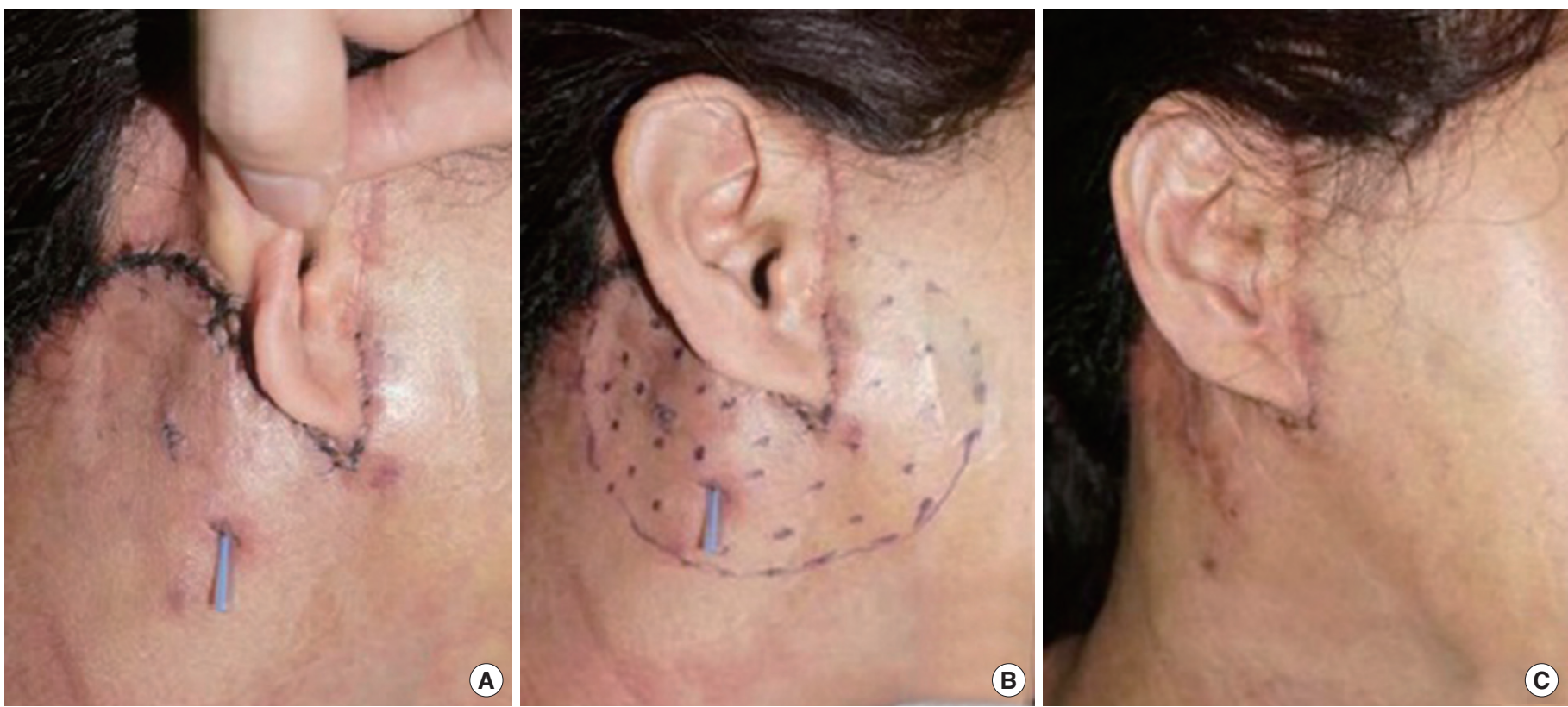

Fig. 2. (A) Lateral view showing swelling and irregular contour of the right side of the preauricular and parotid gland region. (B) Designed lateral view before botulinum toxin injection. (C) Fourteen days after botulinum toxin injection, the patient was free of discomfort, swelling had decreased, and the face contours were symmetrical.

the parotid duct or parenchyma after trauma such as a surgical procedure or facial trauma is one of the major causes of parotid fistula. Extravasation of saliva into glandular and periglandular tissue occurs, leading to significant swelling, infection in the cheek, and abnormal salivary secretion.

Parotid fistula has the potential to resolve spontaneously within 1 month because scar tissue formation occurs around the parotid parenchyma, which prevents further flow of saliva [6]. However, secretion of a large amount of saliva may inhibit healing due to the constant flow of saliva. During the process of delayed wound healing of a parotid fistula, scar contracture may develop, resulting in cosmetic problems. Nevertheless, there are no definite treatment options for parotid fistula. Both surgical and non-surgical approaches have been used to treat parotid fistula.

Conservative approaches include repetition of aspiration, application of a pressure dressing, taking scopolamine, and radiation therapy. However, radiation therapy increases cancer risk, while repetitive aspiration may cause further infections. Continued usage of anticholinergic medication can cause memory problems, urinary retention, and even paralytic ileus $[7,8]$. Surgical procedures such as tympanic neurectomy and marsupialization can also be used to treat parotid fistula after parotidectomy $[9,10]$. However, patients tend to prefer conservative to surgical approaches due to the invasiveness of the latter for cosmetic reasons. Overall, management of a parotid fistula is still controversial.

Botulinum toxin has been shown to have therapeutic effects in patients with sialorrhea. Injection of botulinum toxin into salivary glands is a safe and clinically useful method to treat patients suf- fering from parotid fistula. Botulinum toxin blocks saliva production by inhibiting cholinergic autonomic parasympathetic and postganglionic sympathetic acetylcholine release at the terminal end of the salivary gland.

Botulinum toxin is used to treat various diseases such as sialorrhea and hyperhidrosis [5]. It has also been used to treat drooling associated with Parkinson's disease, pediatric drooling, parotid fistula after parotidectomy, and severe sialorrhea in amyotrophic lateral sclerosis patients or those with neurodegenerative diseases [1, $11,12]$.

However, no previous study has reported injection of botulinum toxin to treat a parotid fistula following facelift surgery.

Several authors have discussed the clinically effective dose of botulinum injection [4,11-14], but there is no consensus regarding dose or method. Various doses have been used; for example 100 units of botulinum toxin type A diluted in $4 \mathrm{~mL}$ of saline, or 2,500 mouse-units of botulinum toxin type B diluted in $4 \mathrm{~mL}$ of saline $[11,12]$. The dose of the toxin has been as high as 100 units at a site, or as low as 10 units at a site. There is also a diversity of injection methods. Reid et al. injected $25 \mathrm{U}$ in the parotid gland and $25 \mathrm{U}$ in the submandibular gland. Laskawi et al. suggested using 10-40 units at two to three sites, while Gregory et al. injected 50 units at two separate sites of the parotid gland for a total dose of 100 units [12-14]. In our study, we used a total of 50 units of intraglandular botulinum toxin type A (Botulex ${ }^{\circledR}$, Hugel, Chuncheon, Korea) diluted in $3 \mathrm{~mL}$ of normal saline, and injected 2 unites at interjunction distances of $1 \mathrm{~cm}$.

We described two patients who complained of excessive sali- 
vary secretion and swelling around the right parotid gland immediately after facelift surgery. Amylase concentration, starch-iodine test results, and sialography results led us to diagnose a parotid fistula in both patients. Instead of performing invasive surgery, we choose to inject botulinum toxin to treat the parotid fistula in both cases. In addition to botulinum toxin injection, patients were instructed to eat rice porridge and minimize temporomandibular joint motion. This is because chewing movements increase salivation from the parotid gland; if mastication is not controlled, then the symptoms of salivary secretion will be exacerbated. Facial bandage and scopolamine were also prescribed. Salivary secretion and swelling subsided effectively and both patients were satisfied with the cosmetic results.

In conclusion, injection of botulinum toxin type A, application of a facial bandage for compression, taking scopolamine, and limiting temporomandibular joint motion is an effective treatment approach for parotid fistulas in facial surgery patients that should be considered before performing an invasive surgical procedure.

\section{REFERENCES}

1. Bushara KO. Sialorrhea in amyotrophic lateral sclerosis: a hypothesis of a new treatment--botulinum toxin A injections of the parotid glands. Med Hypotheses 1997;48:337-9.

2. Winkler E, Goldan O, Regev E, et al. Stensen duct rupture (sialocele) and other complications of the Aptos thread technique. Plast Reconstr Surg 2006;118:1468-71.

3. Baurmash HD. Obstructive parotitis after rhytidectomy. J Oral Maxil- lofac Surg 2004;62:1439.

4. Chow TL, Kwok SP. Use of botulinum toxin type A in a case of persistent parotid sialocele. Hong Kong Med J 2003;9:293-4.

5. Bhidayasiri R, Truong DD. Expanding use of botulinum toxin. J Neurol Sci 2005;235:1-9.

6. Witt RL. The incidence and management of siaolocele after parotidectomy. Otolaryngol Head Neck Surg 2009;140:871-4.

7. Lapid O, Kreiger Y, Sagi A. Transdermal scopolamine use for postrhytidectomy sialocele. Aesthetic Plast Surg 2004;28:24-8.

8. Parekh D, Glezerson G, Stewart M, et al. Post-traumatic parotid fistulae and sialoceles. A prospective study of conservative management in 51 cases. Ann Surg 1989;209:105-11.

9. Westreich M, Binns JH. Posttraumatic parotid pseudocyst with hyperamylasemia. Plast Reconstr Surg 1982;69:1002-6.

10. Habal MB. Parotid retention cysts as a complication of rhytidectomy. Case report. Plast Reconstr Surg 1978;61:920-1.

11. Pantel M, Volk GF, Guntinas-Lichius O, et al. Botulinum toxin type b for the treatment of a sialocele after parotidectomy. Head Neck 2013;35:E11-2.

12. Reid SM, Walstab JE, Chong D, et al. Secondary effects of botulinum toxin injections into salivary glands for the management of pediatric drooling. J Craniofac Surg 2013;24:28-33.

13. Laskawi R, Winterhoff J, Kohler S, et al. Botulinum toxin treatment of salivary fistulas following parotidectomy: follow-up results. Oral Maxillofac Surg 2013;17:281-5.

14. Hatzis GP, Finn R. Using botox to treat a mohs defect repair complicated by a parotid fistula. J Oral Maxillofac Surg 2007;65:2357-60. 